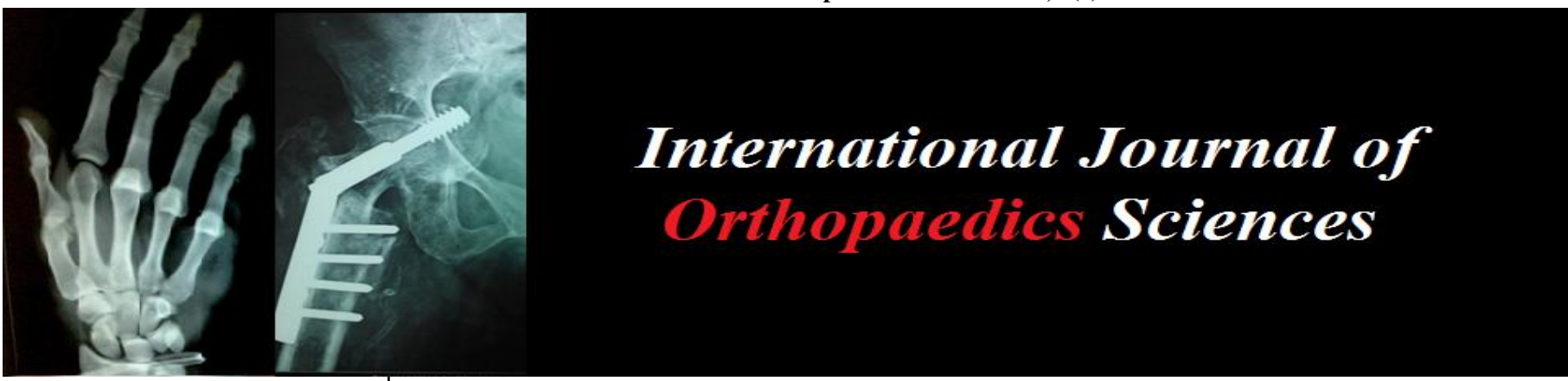

ISSN: $2395-1958$

IJOS 2017; 3(3): 1180-1184

(C) 2017 IJOS

www.orthopaper.com

Received: 10-05-2017

Accepted: 11-06-2017

Handy ED

Department of Surgery and Specialties, Faculty of Medicine and Biomedical Sciences, University of Yaoundé I

Cameroon

\section{Manfo AA}

Department of Surgery and Specialties, Faculty of Medicine and Biomedical Sciences, University of Yaoundé I, Cameroon

\section{Gonsu KH}

Department of Microbiology and Bacteriology, Faculty of Medicine and Biomedical Sciences, University of Yaoundé I, Cameroon

\section{Essi MJ}

Department of Public Health. Faculty of Medicine and Biomedical Sciences, University of Yaoundé I, Cameroon

Ngo Nonga B

Department of Surgery and Specialties, Faculty of Medicine and Biomedical Sciences,

University of Yaoundé I, Cameroon

\section{Sosso MA}

Department of Surgery and Specialties, Faculty of Medicine and Biomedical Sciences,

University of Yaoundé I, Cameroon

\section{Correspondence}

Ngo Nonga B

Department of Surgery and Specialties, Faculty of Medicine and Biomedical Sciences,

University of Yaoundé I,

Cameroon

\section{Clinical and microbiological profile of post-traumatic osteomyelitis in tertiary hospital in Yaoundé - Cameroon}

\author{
Handy ED, Manfo AA, Gonsu KH, Essi MJ, Ngo Nonga B and Sosso MA
}

DOI: $\underline{\text { https://doi.org/10.22271/ortho.2017.v3.i3p.170 }}$

\section{Abstract}

Background: Post-traumatic osteomyelitis is bone infection secondary to a trauma (open fracture or bone surgery). It is a painful and frustrating disease characterised by a high rates of therapeutic failures and a costly management. However, treatment is increasingly becoming difficult because of the phenomenon of antibiotic resistance. The present study was realised to determine the epidemiological and susceptibility profile of bacterial isolates in posttraumatic osteomyelitis in a tertiary hospital in the city of Yaoundé.

Method: A descriptive cross-sectional study was carried out between November 2016 and May 2017. Patients admitted for post-traumatic osteomyelitis were recruited. After recoding clinical informations, deep specimens were taken during surgical debridement and were cultured in Blood, Mannitol Salt and MacConkey plates at $37{ }^{\circ} \mathrm{C}$ for $24-72 \mathrm{hrs}$. Culture isolates were identified by standard biochemical reactions. Antibiotic susceptibility was performed by Kirby-Bauer method using the CASFM guidelines. Results: A total of 31 patients were recruited; the modal age range was $21-50$ years $(67.8 \%)$ and the sex ratio 1.8:4. The commonest bones affected were the tibia (48.5\%) and femur (32.3\%). Predisposing factors identified included open fractures $(76 \%)$, delay surgical debridement $(83.3 \%)$, presence of prosthesis $(58 \%)$ and surgical wound infections (23\%). Out of 31 samples, 29 yielded positive culture giving rise to 48 bacteria isolates. Fourteen samples (48\%) were polybacterial. The most predominant species was Escherichia coli (29\%), followed by Staphylococcus aureus, Pseudomonas aeruginosa and Klebsiella pneumoniae (all 22.6\%). The Gram positive organisms showed good sensitivity to Imipenem, Rifampicin, Fucidine, Lincomycin, and to Vancomycin whereas the Gram negative bacilli were mostly sensitive to Imipenem (96.7\%), Amikacin (82.1\%) and to a lesser extend Quinolones (54\%) and Piperacillin/Tazobactam and Ceftazidime (48\%).

Conclusion: Nosocomial bacteria dominate the bacterial flora of posttraumatic osteomyelitis in our setting and many multidrug resistant strains are emerging thus emphasizing on the importance of hygiene and targeted antibiotherapy.

Keywords: Microbiological profile, Post-traumatic osteomyelitis, antimicrobial susceptibility

\section{Introduction}

Osteomyelitis can be defined as an inflammation of bony tissue caused by an infecting organism. Osteomyelitis can develop from haematogenous spread of an infectious agents, invasion of bone tissue from adjacent site of infection, or direct inoculation of germs following trauma (open fractures or bone surgery) ${ }^{[1]}$. The last entity defines post-traumatic osteomyelitis. The incidence of post-traumatic osteomyelitis is rising in our setting because of the increase in frequency of road traffic accidents and the development of orthopaedic procedures. However, osteomyelitis is a serious and invalidating illness characterised by a high rate of treatment failures and often requires long periods of treatment and hospitalisation leading to temporary impairment and at times long lasting disability or even permanent handicaps ${ }^{[2]}$. Treatment comprises of surgical debridement of all necrotic bone and soft tissue along with use of appropriate antimicrobial therapy. Treatment however is becoming increasingly troublesome due to rise in drug resistant isolates in osteomyelitis cases ${ }^{[3]}$. A good knowledge of the bacterial profile and their sensitivity pattern is therefore essential to initiate a good antibiotherapy while awaiting culture results. 


\section{Materials and Methods}

A descriptive cross-sectional study was carried out from $15^{\text {th }}$ November 2016 to $15^{\text {th }}$ May 2017. Cases of post-traumatic osteomyelitis were recruited at the Orthopaedic ward of the Yaounde Central Hospital (YCH) and after obtaining patient's consent, clinical informations were taken. The important factors taken into consideration were the patient's age, sex, bone involved, signs, symptoms, duration of the illness and predisposing risk factors. Clinical specimens like pus, pus swaps, bone marrow contents and bone sequestrum were then collected during surgery and immediately (within an hour) sent to the Bacteriology Laboratory of Yaoundé University Teaching Hospital (YUTH) for analysis. No specific transport medium was used. The samples were cultured aerobically in Blood, Mannitol salt and MacConkey agar plates. Quality control of the media was done by verifying dates of expiration and the ensuring the absence of growth before usage. The organisms isolated were identified by routine standard operative procedures. Antimicrobial susceptibility testing was done by Kirby-Bauer's disc diffusion method. Antibiotics

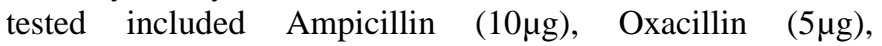
Ticarcilline $(75 \mu \mathrm{g})$, Piperacillin $(30 \mu \mathrm{g})$, Ticarcillin/clavulanic acid $(75 / 10 \mu \mathrm{g})$, Piperacilline/tazobactam (30/6 $\mu \mathrm{g})$, Amoxicillin/clavulanic acid $(20 / 10 \mu \mathrm{g})$, Cefuroxime $(30 \mu \mathrm{g})$, Cefotaxime $(30 \mu \mathrm{g})$, Ceftazidime $(30 \mu \mathrm{g})$, Imipenem $(10 \mu \mathrm{g})$, Vancomycin $(30 \mu \mathrm{g})$, Tobramycin $(10 \mu \mathrm{g})$, Gentamicin $(10 \mu \mathrm{g})$, Amikacin $(30 \mu \mathrm{g})$, Ciprofloxacin $(5 \mu \mathrm{g})$, Levofloxacin $(5 \mu \mathrm{g})$, Erythromycin $(5 \mu \mathrm{g})$, Lincomycin $(15 \mu \mathrm{g})$, Cotrimoxazole

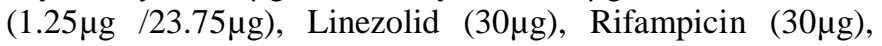
Flucidic acid $(10 \mu \mathrm{g})$. The results wereanalyzed using the 2016 guidelines of the "comité de l'antibiogramme de la société française de microbiologie (CASFM)». Data obtained was entered in CSPro Version 6.3.2 software and transferred into the software SPSS Version 20 for analysis. Results were presented in counts and percentages.

\section{Results}

Thirty one patients with post-traumatic osteomyelitis were admitted in the orthopaedic ward within our study period and were all included in the study. We noted a predominance of the male sex with a sex ratio of 1.8:1. The age group involved majorly was between $31-40$ years $(29 \%)$ followed by $21-30$ years $(25.8 \%), 51-60(19.4 \%), 41-50(12.9 \%)$, and 61 years and above $(12.9 \%)$. The main cause of the primary bone lesion was road traffic accidents, $n=20(65 \%)$ followed by falls, $n=7(21 \%)$ and assault, $n=4(14 \%)$. Surgery was the initial cause of bone exposure in $7(23 \%)$ of the patients against $24(76 \%)$ of patients who had an initial open fracture. Eighteen $(58 \%)$ patients had infections on prosthetic devices. The commonest site affected was the tibia (48.5\%) followed by the femur $(32.3 \%)$ and the ankle $(16.1 \%)$. Twenty four $(83.5 \%)$ of the patients with open fractures were debrided more than 3 days after the initial injury. One (4.2\%) patient was managed within the first 6 hours. $8(26 \%)$ patients first resorted to traditional bone settlers for initial management. Sixteen patients $(52 \%)$ had acute osteomyelitis (symptoms had been evolving for less than 6 weeks) while the remaining $48 \%$ had a chronic bone disease.

Out of the 31 samples analysed, $29(93.5 \%)$ positive culture results were found; $52 \%$ being monobacterial giving a total of 48 bacterial isolates (average of 1.7 bacteria per sample). Gram negative bacteria were the most predominant isolates $(81 \%)$. Enterobacteria represented $62.5 \%$ of the isolates followed by non-fermenting Gram negative bacilli (18.8\%) and Gram positive cocci $(18.8 \%)$. The most predominant species was Escherichia coli with a prevalence of $29 \%$, followed by Staphylococcus aureus, Pseudomonas aeruginosa and Klebsiella pneumoniae (all 22.6\%) (see Table 1).

Table 1: Different bacteria isolated

\begin{tabular}{|c|c|c|c|}
\hline Bacterial group & Species & Number $(\%)$ & Prevalence in patients $(\%)$ \\
\hline Staphylococcaceae $7(14.5 \%)$ & Staphylococcus aureus & $7(14,5 \%)$ & 22.6 \\
\hline & Coagulase negative Staphylococcus & $0(0,0 \%)$ & 0.0 \\
\hline Streptococcaceae $2(4.1 \%)$ & Enterococcus $s p$ & $2(4,1 \%)$ & 6.5 \\
\hline \multirow{9}{*}{ Enterobacteriaceae $30(62.5 \%)$} & Escherichia coli & $9(18,8 \%)$ & 29.0 \\
\hline & Klebsiella pneumoniae & $7(14,5 \%)$ & 22.6 \\
\hline & Enterobacter $s p$ & $4(8,3 \%)$ & 12.9 \\
\hline & Citrobacter sp & $3(6,3 \%)$ & 9.7 \\
\hline & Serratia $s p$ & $2(4,1 \%)$ & 6.5 \\
\hline & Proteus sp & $2(4,1 \%)$ & 6.5 \\
\hline & Morganella morganii & $1(2,1 \%)$ & 3.2 \\
\hline & Kluyvera sp & $1(2,1 \%)$ & 3.2 \\
\hline & Klebsiella terrigena & $1(2,1 \%)$ & 3.2 \\
\hline \multirow{2}{*}{$\begin{array}{c}\text { Non fermenting Gram negative bacilli } \\
9(18.8 \%)\end{array}$} & Pseudomonas aeruginosa & $7(14,5 \%)$ & 22.5 \\
\hline & Pseudomonas fluorescens + putida & $2(4,1 \%)$ & 6.5 \\
\hline
\end{tabular}

All the Staphylococcus aureus strains were sensitive to Imipenem, Rifampicin, Fucidine, Tetracycline, Lincomycin, and to Vancomycin while $50 \%$ were resistant to Oxacillin (MRSA) (figure 1). They all showed reduced sensitivities to the other antibiotics. The two strains of Enterococcus sp. isolated were susceptible to most of the antibiotics except for Gentamicin, Erythromycin and Oxacillin for which only 1 strain was sensitive. The different isolates of Pseudomonas $s p$. were most sensitive to Imipenem (88.9\%), Amikacin
(75\%) and Quinolones (66.7\%). Only about one half (55.6$57 \%$ ) of the isolates were sensitive to Ticarcilline-clavulanic acid and Piperacillin-tazobactam (figure 2). A few antibiotics inhibited the growth of the majority of the enterobacteria isolates. These included Imipenem (97\%), Amikacin (82\%) and Levofloxacin/Ciprofloxacin (54\%) and to a lesser extent, Piperacillin-Tazobactam (48\%), Ceftazidime (43\%) and Ticarcillin-clavulanic acid (38\%) (See figure 3). 


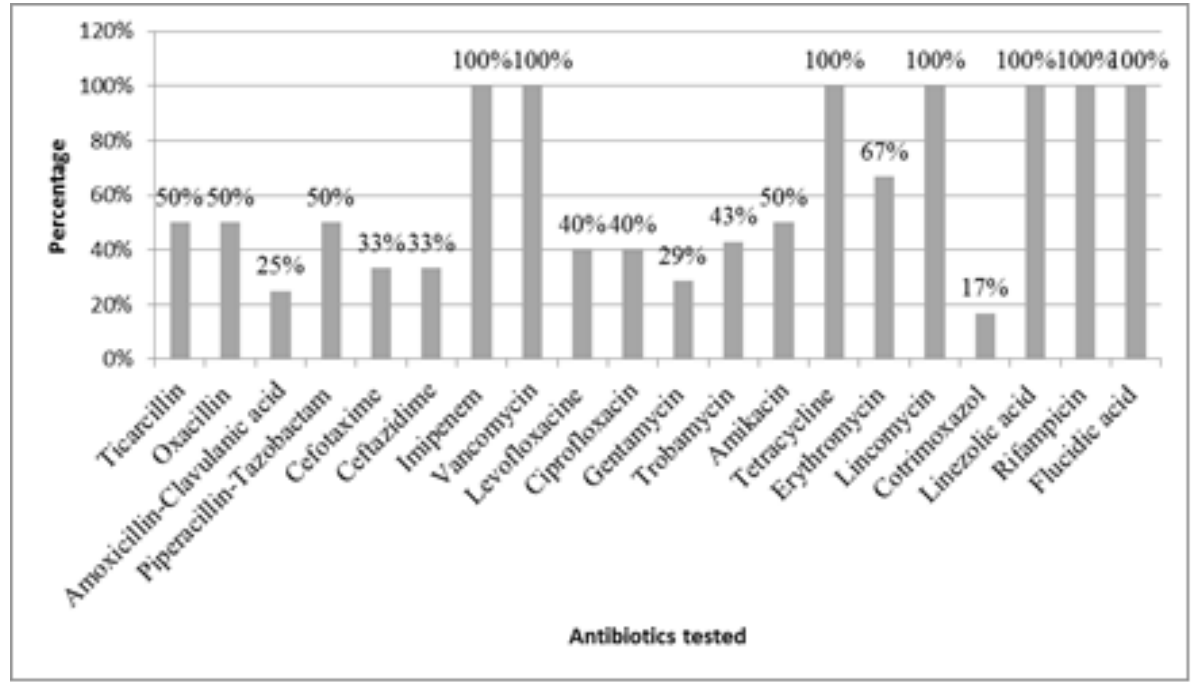

Fig 1: Antimicrobial Sensitivity pattern of Staphylococcus aureus

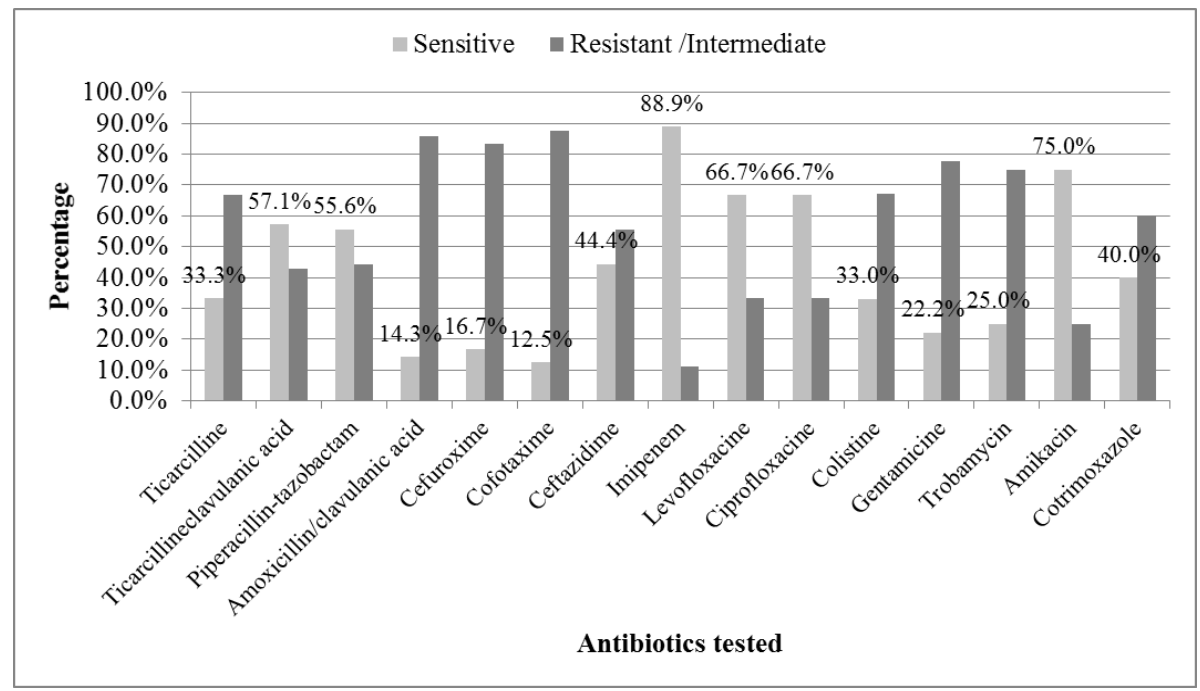

Fig 2: Sensitivity profile of Pseudomonas sp.

\section{Discussions}

\section{Clinical profile}

The incidence of posttraumatic osteomyelitis was observed high among males (with a sex ratio of 1.8:1) and in the age groups between 31-40 (29\%) and 21-30 (25.8\%). This high predominance of the male gender and the youthful age group could be attributable to the greater likelihood of trauma and compound fractures in this population group in relation with their daily activities. These results are similar to those obtained in a study done in India in 2017 also reported a higher cases of osteomyelitis among younger age groups of $31-40(32.8 \%)$ followed by $21-30(25.6 \%)^{[4]}$. A study in Brazil, 2012 reported similar results ${ }^{[5]}$.

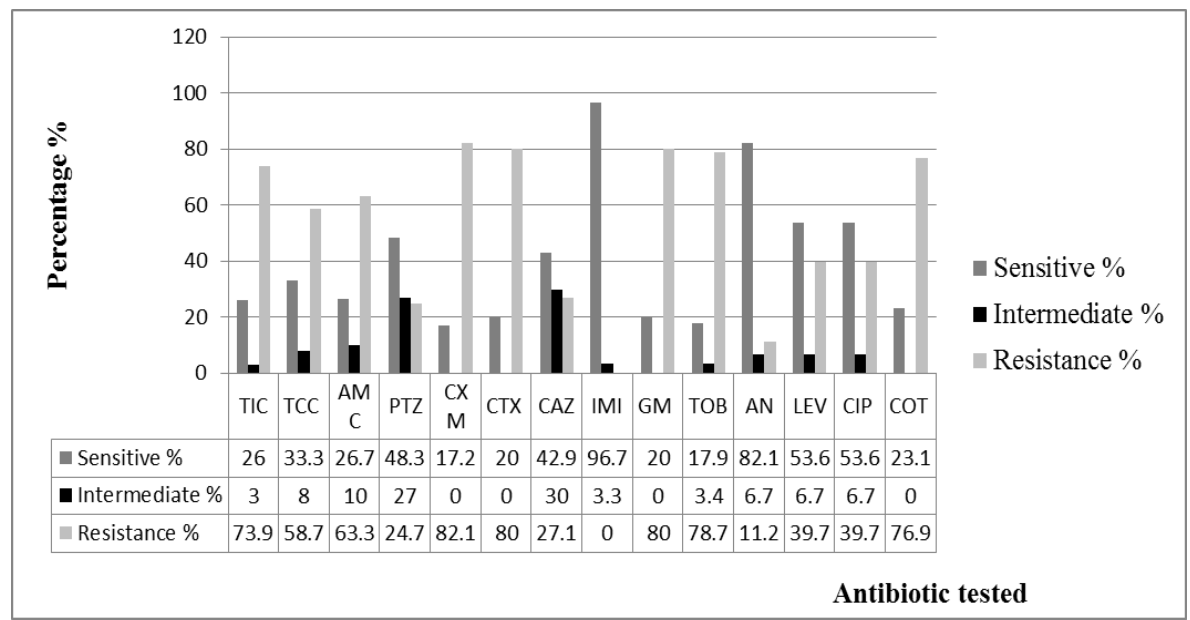

Fig 3: Antimicrobial susceptibility pattern for enterobacteria $\mathrm{ATB}=$ Antibiotic $\mathrm{S}=$ Sensitive $\mathrm{I}=$ Intermediate $\mathrm{AMC}=\mathrm{Amoxicillin}$ Clavulanic acid $\mathrm{CAZ}=$ Ceftazidime $\mathrm{CTX}=$ Cefotaxime $\mathrm{GM}=$ Gentamicin COT= Cotrimoxazole LEV= Levofloxacin CIP= Ciprofloxacin CXM=Cefuroxime $\mathrm{AN} / \mathrm{A}=$ Amikacin $\mathrm{TOB}=$ Tobramycin TCC $=$ Ticarcillin/Clavulanic PTZ= Piperacillin Tazobactam TIC $=$ Ticarcillin IMI $=$ Imipenem 
Road traffic accidents were the major cause of primary bone injury in our study accounting for up $65 \%$ of the causes. The advent of motor cycles, the nature of roads in our setting and the non-respect of road security measures may explain these findings ${ }^{[6]}$. The next predominant cause of injury was falls (21\%). Most of the cases of falls however were elderly women who are known to have reduced bone density associated with their menopausal status. These results are similar to those obtained in other studies in African context ${ }^{[7}$, ${ }^{8}$. Of these bone lesions, $76.7 \%$ of the patients developed the bone infection after an initial open fracture. Open fractures expose the bony tissue thereby increasing the risk of inoculation of bacteria into the bone either during the accident or during wound dressing at the hospital [9]. This was even more evident in our study as almost all $(96 \%)$ of the patients were debrided after 6hours; $83 \%$ being debrided after 3 days, when local signs of infection had started appearing. Twenty six percent of the patients resorted to traditional bone settlers for initial management. A retrospective study done in Mali from 1994-2003 reported that 57\% of the patients first consulted traditional doctors before consulting at hospitals ${ }^{[10]}$. This constant resort to traditional bone settler could be explained by ignorance and poverty in our setting. Postsurgical wounds were the predisposing factor in $23.3 \%$ of the patients in our study. These were probably a consequence of defects in the respect of hygienic conditions during surgery or wound dressing. Metal works (implants) were associated with $58 \%$ of infections. In our study, predominant involvement of the lower limb was noticed with the tibia, the femur and bimalleolar accounting for $48.5 \%, 32.3 \%$ and $16.1 \%$ respectively. The tibia because of its anatomic situation (proximity to the skin) is the bone that is most susceptible to open fractures and therefore to osteomyelitis.

In a study realized in India in $2013^{[11]}$, the major predisposing factors identified included open fractures $(53 \%)$, postoperative infections (26\%) and implants (19\%). The most affected bones were the same as for our study and included the tibia $(58 \%)$ and the femur $(31 \%)$. Another study realized in India in $2016^{[4]}$ identified as predisposing factors open fractures $(45.6 \%)$, post-operative infections (27.2\%) and implants $(24.0 \%)$. The most affected bones also included tibia and the femur $(44.0 \%$ and $40.8 \%$ ) respectively. The higher percentage of open fractures in our study could be explained by the fact that we only considered post traumatic osteomyelitis meanwhile those studies also included haematogenous osteomyelitis and osteomyelitis on diabetic foot ulcers. With respect to duration of illness, 52\% of our patients had an acute infection while $48 \%$ had a chronic infection. In the study done in India in $2016,77.8 \%$ of the patients had chronic osteomyelitis in contrast to $22.2 \%$ who had an acute infection. However, they included in their study haematogenous osteomyelitis and diabetic foot ulcers in their study.

\section{Culture results}

In our samples, $93.5 \%$ gave positive culture results and enabled the isolation of a total of 48 isolates giving an average of 1.4 bacteria per sample. Overall, $52 \%$ of the samples were monobacterial while $48 \%$ were polybacterial. Our results are similar to those reported in a similar study done in Marocco, in 2007, which reported the average of 1.4 bacteria per specimen and $63.5 \%$ were monomicrobial against $36.5 \%$ of the cases which were polymicrobial ${ }^{[12]}$. The study realized in India in 2010 reported $72 \%$ of positive cultures, $70 \%$ of these being monobacterial cultures and $30 \%$ polybacterial ${ }^{[13]}$. In our study, Gram negative bacilli were the most common bacteria isolated (62.5\%) with Escherichia coli being the most predominant organism $(18.8 \%)$ followed by Staphylococcus aureus, Pseudomonas aeruginosa and Klebsiella pneumoniae (14.5\%). This corresponds to the profile of nosocomial germs showing the latter a major role in posttraumatic osteomyelitis in our context. This does not correlate with most studies as Staphylococcus aureus is often reported to be the first causative agent ${ }^{[4,11,14,15]}$. A similar study done in Iraq in 1998 however reported Pseudomas sp, Klebsiella $s p$ and Proteus $s p$ to be more predominant than Staphylococcus aureus ${ }^{[16]}$.

\section{Sensitivity testing}

All the strains of Staphylococcus aureus were sensitivity to Imipenem, Rifampicin, Tetracycline, Flucidic acid, Lincomycin and to Vancomycin (VSSA) while 50\% were susceptible to Oxacillin (MSSA). Their sensitivity for quinolones, aminoglycosides, Cotrimoxazole and betalactams (including combination forms with betalactamase inhibitors like Amoxicillin-Clavulanic acid) was generally low (less than 50\%). These findings are different from those obtained in the study carried out in Morocco $2014^{[12]}$ which reported a good (87-100\%) sensitivity of all the Staphylococcus aureus strains to most antibiotics except for Penicillin G. All the strains were methicillin and Vancomycin sensitive. The difference could be explained by the fact that they mostly had community acquired germs. The studies carried in India in $2013^{[11]}$ and $2017^{[4]}$ had comparable results with ours. They showed reduced sensitivity (less than 50\%) to quinolones, aminoglycosides, Cotrimoxazole and to Cephalosporins. The percentages of MSSA were $56.9 \%$ and $76.1 \%$ respectively and all samples in both studies were however sensitive to Vancomycin. The overall sensitivity of the strains of Enterococcus $s p$. were generally high correlating with the findings in a similar study done in India in $2016^{[15]}$.

The isolates of Pseudomonas sp. were most sensitive to Imipenem (88.9\%), Amikacin (75\%) and Quinolones (66.7\%) followed by Ticarcilline-clavulanic acid and Piperacillintazobactam (55.6 and 57\% respectively). They showed reduced (12.5-25\%) sensitivities to the other aminoglycosides, Amoxicillin-clavulanic acid and cephalosporins. The study in India $2013^{[11]}$, reported a best sensitivity rates to be amongs Piperacillin-Tazobactam (89\%), Amikacin (78\%) and the Quinolones (67-89\%). The sensitivity to cephalosporins was also low but that to aminoglycosides relatively higher (67\%). These findings also correlate to the 2003 report of "The Surveillance Network, TSN "USA [17]. The study in Morocco 2014 reported a good (more than 50\%) sensitivity to most antibiotics except for Amoxicillin-Clavulanic acid, Ampicillin and Cefalotin ${ }^{[12]}$.

Most of the Enterobacteria in our study were sensitive to Imipenem (97\%), Amikancin (82\%) and quinolones (54\%) and about $50 \%$ were either sensitive or intermediate to Piperacillin/Tazobactam, and Ceftazidime. They showed high resistance to aminoglycosides, other cephalosporins, and Cotrimoxazole. This susceptibility rate is relatively low compared to the findings of "The Surveillance Network, TSN "USA which reports high sensitivities $(>78 \%)$ of the different enterobacteria strains to quinolones, third generation cephalosporins, and piperacillin/Tazobactam [17]. This difference could be explained by the fact that most of these organisms in our study were probably hospital acquired. The findings however correlate with those of the study done in India in $2013^{[11]}$ where a higher sensitivity among 
enterobacteria was noted only to Imipenem, Amikacin and Quinolones. Another study done in India, 2014 on chronic osteomyelitis reported even lower rates sensitivities (less than $50 \%$ ) for most antibiotics except Imipenem and Amikacin.

However, while antimicrobial therapy is desirable in the control of osteomyelitis, the most important factor for a successful treatment of patients with bone infection is the quality of debridement. The debridement should be done early enough and must achieve a clean and viable wound. Appropriate therapy of posttraumatic osteomyelitis includes adequate drainage, thorough debridement, obliteration of dead space, stabilization when necessary, wound protection, and specific antimicrobial therapy.

\section{Conclusions}

Nosocomial bacteria dominate the bacterial flora of posttraumatic osteomyelitis in our setting and these bacteria are developing resistances to most of the routinely used antibiotics. Appropriate implementation of hygienic practices and appropriate selection of antibiotics based on cultures results will help treat the disease successfully in the early stages and prevent the spread of multidrug-resistant strains to limit morbidity.

\section{Acknowledgement \\ None}

\section{Ethical considerations}

The manuscript has received approval from the ethical committee of the Faculty of Medicine and biomedical sciences of the University of Yaoundé I, Cameroon

\section{References}

1. Seong Yeol Ryu RP. Chapter 2: Microbiology of bone and joint infections. In: Werner Z, editor. Bone Jt. Infect. From Microbiol. to Diagnostics Treat. First Edit. John Wiley \& Sons, Inc; 2015, 5-17.

2. Poultsides LA, Liaropoulos LL, Malizos KN. The socioeconomic impact of musculoskeletal infections. J Bone Joint Surg Am. 2010; 92:1-12.

3. Nathan C, Cars O. Antibiotic resistance-problems, progress, and prospects. N Engl J Med 2014; 371:1761-3.

4. Khatoon R, Khan SA, Jahan N. Antibiotic resistance pattern among aerobic bacterial isolates from osteomyelitis cases attending a Tertiary care hospital of North India with special reference to ESBL, AmpC, MBL and MRSA production. Int J Res Med Sci. 2017; 5:482-90.

5. Carvalho V, Rosalba P, Oliveira D, Karine DP, Paula AP. Gram-negative osteomyelitis: clinical and microbiological profile. Brazilian J. Infect. Dis. 2012; 16:63-7.

6. Ngaroua D, Neossi N, Amvene JM, Mefire AC, Eloundou N. Epidemiology and pattern of road traffic injuries in Ngaoundéré, Cameroon: a retrospective hospital based study prior to the implementation of a formal trauma registry. J Med. Heal. Sci. 2014; 15:1-8.

7. Ngim N, Udosen A, Ikpeme ON I. Prospective Study of Limb Injuries in Calabar. Internet J. Orthop. Surg. 2007, 8.

8. Adeleke Olusegun Ifesanya, Temitope Oluwagbenga Alonge, Samuel Olusegun Ogunlade, Ajibade B Omololu, Timothy Eyo Nottidge ROA. Changing trends in the pattern of tibial fractures in Nigeria: A review of 70 cases. J. Orthop. 2008; 5:e2.
9. Jedlicka N, Summers JN, Murdoch MM. Overview of concepts and treatments in open fractures. Clin. Podiatr. Med. Surg. 2012; 29:280-90.

10. Sangare A, Alwata I, Sidibe S, Macalou M, TAA. Les ostéites dans le service d'orthopédie et de traumatologie de l'hôpital Gabriel Toure de Bamako. MALI Med. 2008; $1: 27-30$.

11. Suguneswari G, Singh AH, Basu R. Bacteriological profile of osteomyelitis in a tertiary care hospital at Visakhapatnam, Andhra Pradesh. Andhra Pradesh Int J Cur Res Rev. 2013; 5:52-8.

12. Elouennass M, El Hamzaoui S, Frikh M, Zrara A, Chagar B, Ouaaline M. Les aspects bactériologiques des ostéites dans un hôpital universitaire. Médecine Mal. Infect. 2007; 37:802-8.

13. Sheehy SH, Atkins BA, Bejon P, Byren I, Wyllie D, Athanasou NA et al. The microbiology of chronic osteomyelitis: prevalence of resistance to common empirical anti-microbial regimens. J. Infect. 2010; 60:338-43.

14. Coudane H, Gérard A, George T, Claudot F, Gervaise A. Ostéites. Encycl. Medico-Chirurgical. 2011, 1-14.

15. Gopi A, Misbah S, Khair U, Kottileveetil HT, Harindranath D, Sabapathy V. A clinico-microbiological study of osteomyelitis in a tertiary care hospital in Karnataka. J Evol. Med Dent Sci. 2016; 5:15-8.

16. Mousa HAL. Post-traumatic bone infection. Qatar Med. J. 1998; 7:44-5.

17. Jones ME, Karlowsky JA, Draghi DC, Thornsberry C, Sahm DF, Nathwani D. Antibiotic susceptibility of bacteria most commonly isolated from bone related infections: the role of cephalosporins in antimicrobial therapy. Int. J Antimicrob. Agents. 2004; 23:240-6. 